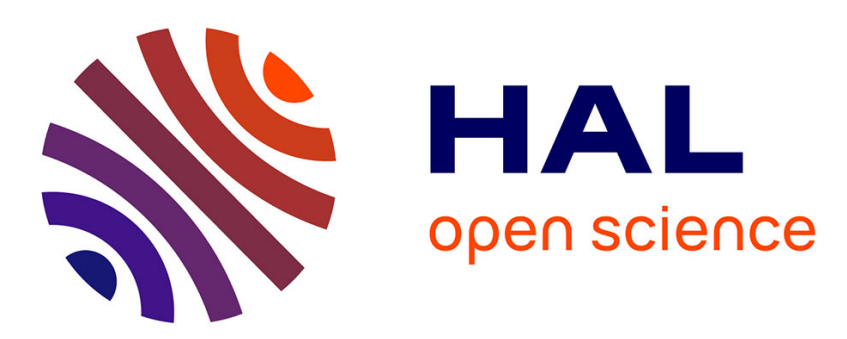

\title{
Bien vieillir et bien-être : le rôle des objets connectés dans l'activité physique des seniors
}

\author{
Agnès Helme-Guizon, Romain Debru
}

\section{To cite this version:}

Agnès Helme-Guizon, Romain Debru. Bien vieillir et bien-être: le rôle des objets connectés dans l'activité physique des seniors. Décisions Marketing, 2020, 98, pp.15-36. 10.7193/DM.098.15.36 . hal-03160510

\author{
HAL Id: hal-03160510 \\ https://hal.science/hal-03160510
}

Submitted on 5 Mar 2021

HAL is a multi-disciplinary open access archive for the deposit and dissemination of scientific research documents, whether they are published or not. The documents may come from teaching and research institutions in France or abroad, or from public or private research centers.
L'archive ouverte pluridisciplinaire HAL, est destinée au dépôt et à la diffusion de documents scientifiques de niveau recherche, publiés ou non, émanant des établissements d'enseignement et de recherche français ou étrangers, des laboratoires publics ou privés. 


\title{
Bien vieillir et bien-être : le rôle des objets connectés dans l'activité physique des seniors
}

\author{
Agnès Helme-Guizon ${ }^{*}$ et Romain Debru** \\ *Univ. Grenoble Alpes, Grenoble INP (Institute of Engineering), CERAG et Grenoble IAE \\ **Univ. Grenoble Alpes, Ecole Doctorale de Sciences de gestion (EDSG), Laboratoire CERAG
}

Résumé : Pratiquer une activité physique est bénéfique pour la prévention ou le traitement de nombreuses maladies et participe donc au bien vieillir. Néanmoins, initier et maintenir ce comportement chez les seniors semble difficile. Cette étude, ancrée dans la théorie de l'AutoDétermination, a pour objectif d'étudier l'activité physique quotidienne des seniors retraités et de montrer le rôle joué par les objets connectés, tout particulièrement sur leur motivation à s'engager durablement dans une activité physique. L'analyse des données collectées auprès de 21 retraités âgés de 63 à 82 ans au moyen d'entretiens semi-directifs (14 participants) et deux mini-groupes ( 3 et 4 participants) met en évidence trois profils : les Contraints, les Attentifs et les Engagés. Ils varient dans la satisfaction des besoins psychologiques fondamentaux, le type de motivation, la durabilité de la pratique d'activité physique ainsi que la perception des objets connectés et de leur rôle dans la pratique de l'activité physique. Ces profils sont discutés et des recommandations sont proposées.

Mots clés : Seniors ; Activité physique ; Théorie de l'auto-détermination ; Objets connectés ; Bien vieillir ; Bien-être

\begin{abstract}
Physical activity provides benefits for the prevention or treatment of many diseases and thus contributes to aging well. Nevertheless, initiating and maintaining this behaviour over the long term seems difficult, especially among seniors. This study, grounded in the theoretical framework of Self-Determination Theory, aims at studying retired older adults physical activity practices and at showing the role played by connected objects peculiarly on their motivation to engage sustainably in physical activity. Twenty-one respondents aged 63 to 82 years participated in the study. The analysis of the data collected through semi-directive interviews (14) and 2 minigroups ( 3 and 4) lead to a qualitative typology organized around three profiles: the Compelled, the Vigilent and the Committed. They vary in the satisfaction of the basic psychological needs, the type of motivation, the sustainability of physical activity practice as well as the perception of smart devices and the role they plan in physical activity. These profiles are discussed and recommendations are provided.
\end{abstract}

Keywords: Seniors; Physical Activity; Self-determination Theory; Smart Devices ; Aging Well; Well-Being 
La population française vieillit. En 2017, les plus de 65 ans représentaient 19,2\% de la population ${ }^{1}$. En 2050, ils devraient être plus de 20 millions (soit 8,6 millions de plus qu'en 2013) et atteindre $27,2 \%$ de la population. Ces personnes de plus de 65 ans sont considérées comme des seniors... au même titre que les 50 ans et plus. De fait, des divergences existent quant à la limite inférieure de ce groupe (Urien, 2014). Afin de ne pas entrer dans ce débat, nous faisons le choix de nous centrer sur les retraités de plus de 60 ans pour cette étude. Le passage à la retraite est une étape importante de la vie et peut être vécue comme une rupture avec le monde « actif» (Arbuz, 2013). Dans tous les cas, c'est une transition qu'il convient de gérer, notamment par la réorganisation de son mode de vie en lien avec le temps libéré et les conséquences du vieillissement.

Le vieillissement est un processus physiologique normal qui s'accompagne d'un déclin cognitif, d'une perte de capacités physiques et d'une augmentation du risque de chutes qui conduit in fine, à une perte d'autonomie (Mareschal et Genton, 2014). L'avancée en âge est également un facteur de risque pour l'apparition de diverses pathologies (e.g., cancer, maladies cardiovasculaires, Mareschal et Genton, 2014). Dans un contexte de vieillissement de la population, la question du bien vieillir devient alors légitime. Actuellement, deux conceptions du bien vieillir co-existent. L'approche biomédicale l'évalue à l'aide de critères comme l'absence de maladie, de handicap, de facteurs de risque, la préservation du fonctionnement physique et cognitif ou encore l'implication dans des activités sociales. L'approche subjective définit le bien vieillir comme « une adaptation continue des objectifs personnels et des comportements visant à minimiser les pertes et à maximiser les gains liés à l'avancée en âge " (Sengès et al., 2018). Dans cette recherche, nous adoptons l'approche subjective qui reste majoritairement utilisée en marketing. Le bien vieillir représente trois enjeux majeurs. Le premier est un enjeu de santé publique, visant à réduire le nombre de maladies et de prises en charge. En effet, la proportion des personnes ayant des maladies chroniques augmente avec l'âge (15\% pour les 15-24 ans, 49\% pour les 55-64 ans et $76 \%$ pour les personnes âgées de 85 ans et plus) $)^{2}$. Le second est un enjeu financier car ces maladies et les différents traitements représentent un coût très élevé. Enfin, le troisième est un enjeu de marketing : le vieillissement étant un processus hétérogène, il convient de proposer des biens et services adaptés aux besoins spécifiques des seniors (Pierre et al., 2015). Il est important de mieux comprendre la perception des seniors sur ce vieillissement et les stratégies mises en place pour contrer ses effets. La prise en compte de ces spécificités a fait naître la Silver

\footnotetext{
${ }^{1}$ https://www.insee.fr/fr/statistiques/3197277?sommaire=3197289; accédé le 4/12/2017.

2 Direction de la recherche des études de l'évaluation et des sta- tistiques. L'état de santé de la population en France. Suivi des objectifs annexés à la loi de santé publique. Rapport 2011. Paris: DREES; 2011.
} 
Economy, définie comme "l'économie dédiée à l'avancée en âge de nos sociétés » De nombreux secteurs économiques sont concernés (e.g., santé, finance, bien-être, technologies, alimentation, etc.). Elle se traduit par l'offre de produits et de services dédiés spécifiquement aux seniors dans le but de préserver leur autonomie aussi longtemps que possible (Vassli et Farshchian, 2018). L'objectif étant de contribuer à leur bien-être qui « réside dans un sentiment général d'agrément, d'épanouissement suscité par la pleine satisfaction des besoins du corps et/ou de l'esprit » (Guibet Lafaye, 2007) et de manière générale à leur bonne santé ; c'est-à-dire « un état de complet bien-être physique, mental et social, et ne consiste pas seulement en une absence de maladie ou d'infirmité $»^{4}$ selon la définition de l'Organisation Mondiale de la Santé (OMS).

Les effets du vieillissement peuvent être retardés, entre autres, par la pratique régulière d'une activité physique c'est-à-dire par «tout mouvement produit par les muscles squelettiques, responsable d'une augmentation de la dépense énergétique $»^{5}$. Elle ne doit pas être confondue avec le sport qui renvoie à des notions de participation organisée et de compétition ${ }^{6}$. Les nombreux bénéfices d'une activité physique sur la santé physique mais également mentale des personnes (Mareschal et Genton, 2014) ainsi que sur la création et le maintien de relations sociales (Barth et al., 2018) ne sont plus à démontrer. Les personnes de plus de 65 ans peu actives ont un taux de mortalité, toutes causes confondues, supérieur à leurs homologues plus actifs physiquement ${ }^{7}$. Différentes initiatives ont été prises pour promouvoir la pratique d'une activité physique régulière chez les seniors : le plan national Bien Vieillir ou encore le Plan National de Prévention de la Perte d'Autonomie. Récemment, a été votée la loi sur la prescription de séances d'Activité Physique Adaptée (APA) par le médecin (Barth et al., 2018). L'OMS a également établi des recommandations en matière d'activité physique pour les seniors ${ }^{7}$. Toutefois, ces initiatives montrent leurs limites. Par exemple, en ce qui concerne la recommandation des 10.000 pas quotidiens, l'étude réalisée par l'association Attitude Prévention montre que $76 \%$ des Français âgés de 18 à 64 ans sont en dessous de cette recommandation avec une moyenne de 7 889 pas par jour. Le constat est encore plus alarmant chez les plus de 65 ans avec une moyenne de 5144 pas quotidiens ${ }^{8}$. Bien que les bénéfices de l'activité physique soient clairement établis, seuls $40 \%$ des seniors entre 55 et 75 ans atteignent les niveaux de recommandations de santé

\footnotetext{
${ }^{3}$ https://www.silvereco.fr/silver-economy; Consulté le 20/02/2019.

${ }^{4}$ https://www.who.int/suggestions/faq/fr// Consulté le 20/01/2019.

${ }^{5}$ https://www.who.int/dietphysicalactivity/pa/fr/ ; Consulté le 20/01/2019.

${ }^{6} \mathrm{http}$ ://www.sports.gouv.fr/pratiques-sportives/sante-bien-etre/Donnees-scientifiques/; Consulté le 17/09/2018.

7 https://www.who.int/dietphysicalactivity/factsheet olderadults/fr/ ; Consulté le 19/02/2019.

8 www.attitude-prevention.fr ; Accédé le 04/12/2017.
} 
publique en matière d'activité physique ${ }^{9}$. Dès lors, se pose la question sur la manière d'engager et/ou maintenir durablement les seniors dans une activité physique régulière. Se basant sur la théorie de l'auto-détermination (Deci et Ryan, 1985) selon laquelle la satisfaction des trois besoins psychologiques fondamentaux (autonomie, compétence et affiliation) détermine le niveau et le type de motivation, cette recherche s'intéresse à la motivation autonome comme levier de l'engagement et du maintien d'une activité physique, source de bien vieillir et de bien-être (Ryan et al., 2008).

De plus, face au foisonnement d'offres d'objets connectés (e.g., montres, trackers d'activité, alarmes, détecteurs de chute, balances ou tensiomètres connectés, etc.) proposés aux seniors par la Silver Economy pour les aider à prendre soin de leur santé et à s'engager dans une activité physique, cette recherche s'interroge sur la manière dont ces objets connectés sont perçus et utilisés par les seniors, sur leur place dans la pratique d'une activité physique, leur impact sur leur motivation et in fine leur rôle sur le bien vieillir et le bien-être des seniors.

Ces questions, à notre connaissance, n'ont pas ou peu été explorées par la recherche en marketing. L’hétérogénéité des pratiques ainsi que leur rapport aux objets connectés (Pierre et al., 2015) rend nécessaire une typologie qualitative de seniors, fondée sur leur niveau d'activité physique, leur auto-détermination, leur motivation et leur relation aux objets connectés.

Les contributions de notre recherche sont nombreuses. Tout d'abord, elle propose la théorie de 1'auto-détermination comme un cadre théorique ${ }^{10}$ permettant d'expliciter les facteurs sous-jacents aux différents niveaux d'activité physique des seniors et en montre la pertinence sur ce public. Ensuite, elle fait explicitement le lien entre la pratique d'activité physique et l'utilisation d'objets connectés chez les seniors, ce qui est à notre connaissance novateur. La construction d'une typologie de seniors (basée sur leur niveau d'activité physique, leur motivation et leur rapport aux objets connectés) ainsi que les recommandations qui en découlent, viennent directement enrichir la littérature existante. De plus, cette recherche contribue au courant du bien vieillir en mettant en exergue le rôle que peut jouer les objets connectés pour amener les seniors vers une motivation autonome, levier de pérennisation d'une activité physique régulière. Enfin, elle contribue à l'agenda de recherche sur les consommateurs âgés et les technologies (Nunan et Di Domenico, 2019).

\footnotetext{
${ }^{9}$ Escalon H., Bossard C. et Beck F. (dir.) (2009) Baromètre santé nutrition 2008 ; coll Baromètres santé, 424 p

${ }^{10}$ Au-delà du marketing, un tel cadre est fortement demandé par l' Inserm. "L'identification des facteurs psychologiques associés à la pratique d'une activité physique est cruciale dans une perspective d'intervention visant à promouvoir l'engagement des personnes âgées.”, rapport d'expertise collective, ch.9, p.275, Inserm, http://www.ipubli.inserm.fr/bitstream/handle/10608/6807/?sequence=17; accédé le 28/10/2019.
} 


\section{L'auto-détermination, levier d'engagement dans l'activité physique}

Parmi les facilitateurs de l'engagement dans l'activité physique, la motivation représente un levier important pour la pratique d'une activité physique régulière (Sarrazin et al., 2016 ; Teixeira et al., 2012). La théorie de l'auto-détermination est une théorie de la motivation qui s'inscrit dans une vision organismique. Elle postule que l'individu est un organisme actif et évolutif, qui cherche à se développer et à intégrer de nouvelles expériences cohérentes avec ses valeurs (Deci et Ryan, 1985). D’une grande puissance heuristique, elle intègre les antécédents, processus et conséquences de la motivation ainsi que des modérateurs (Gillet et Vallerand, 2016). Elle permet ainsi une approche holistique du comportement. Elle a été testée empiriquement dans une variété de domaines (santé, travail, éducation), dont l'activité physique (Sarrazin et al., 2016).

La théorie de l'auto-détermination suggère que le niveau de motivation d'une personne dépend du niveau de satisfaction de trois besoins psychologiques fondamentaux (autonomie, compétence, affiliation). Le besoin de compétence correspond au fait de se sentir efficace dans ses interactions avec l'environnement. Le besoin d'autonomie désigne le fait d'être l'origine ou la source de ses propres comportements, d'agir selon sa propre volonté et son libre choix. Le besoin d'affiliation concerne la recherche des sentiments d'intimité, de sécurité et de connexion avec un groupe social que l'individu juge important (donner / recevoir de l'attention) (Deci et Ryan, 2000). Il est courant de distinguer trois grands groupes de motivation : l'amotivation, la motivation contrôlée et la motivation autonome qui se répartissent sur un continuum. Elles induisent des degrés croissants de durabilité des comportements (ici l'engagement dans l'activité physique) et de bienêtre (Deci et Ryan, 2016 ; Ryan et al., 2008). L'annexe 1 détaille les types de motivation ainsi que les mécanismes sous-jacents de régulation (intégration des facteurs externes et internes).

\section{Les seniors et l'activité physique}

La motivation des seniors pour la pratique d'une activité physique

Avec l'avancée en âge, la satisfaction des trois besoins psychologiques fondamentaux est modifiée. La diminution des capacités physiques entraîne une baisse de l'autonomie de la personne mais également de son sentiment de compétence, et donc de sa motivation à faire des activités. De plus, voir son conjoint ou ses amis mourir, affecte son besoin d'affiliation. A l'inverse, une amélioration de la satisfaction de ces trois besoins est liée positivement à une amélioration de la santé des seniors (Custers et al., 2010). Plus spécifiquement, deux études ont montré le rôle de la motivation dans la pratique d'une activité physique chez les seniors. Dacey et al. (2008) ont évalué les motivations à l'égard de l'activité physique de 645 hommes (âge moyen 
de 63,8 ans). Ils montrent que le type de motivation est un bon indice pour discriminer le niveau d'activité physique pratiquée par les seniors (inactif, actif et très actif). Une motivation intrinsèque, au sens de la théorie de l'auto-détermination, est positivement liée à un niveau élevé d'activité physique. Le plaisir que procure l'activité physique est également un bon prédicteur du type de motivation et de la quantité d'activité physique pratiquée. Par ailleurs, Ferrand et al. (2012) sur un échantillon de 92 sujets âgés en moyenne de 74,95 ans, ont mis en évidence deux groupes. Le premier groupe (actif) possédait un haut niveau de motivation intrinsèque et de régulation identifié qui correspondent à des types de motivation autonomes et favorables au changement de comportement. Le second groupe (moins actif) possédait un faible niveau d'autodétermination avec une motivation extrinsèque et une régulation introjectée. Ces deux études soulignent la pertinence de la théorie de l'auto-détermination pour identifier et comprendre la motivation des seniors à s'engager et maintenir une activité physique.

\section{L'activité physique, une clé du bien-être et du bien vieillir}

Le bien-être résulte de la satisfaction des besoins psychologiques fondamentaux et sera d'autant plus important que la motivation est autonome (Ryan et al., 2008). Augmenter ou maintenir le sentiment d'autonomie et de compétence des seniors produit des effets favorables sur leur bienêtre ainsi que sur leur santé physique et mentale (Paquet et al., 2016). De nombreuses études ont montré l'effet positif de la satisfaction des besoins psychologiques fondamentaux dans le cadre d'une activité physique et de la motivation autonome (vs contrôlée) à pratiquer une activité physique sur le bien-être (pour une revue, voir Sarrazin et al., 2016).

Le vieillissement étant inéluctable, autant le vivre dans les meilleures conditions possibles. Selon l'approche subjective du bien vieillir, le bien-être physique et psychologique font partie intégrante de celui-ci. En effet, en retardant le déclin cognitif lié à l'âge, la pratique régulière d'activité physique va permettre aux seniors de maintenir leur autonomie, et de ce fait leur qualité de vie (Mareschal et Genton, 2014). Il est important de conserver les capacités fonctionnelles des seniors (i.e., marche, préhension, force musculaire). L'activité physique a donc une action «protectrice » sur le bien-être physique des seniors. Quant au bien-être psychologique, être satisfait de sa vie actuelle et passée et se sentir heureux, participe au bien vieillir. Redonner du contrôle aux seniors et les rendre actifs de leur vieillissement est la clé du bien vieillir.

La motivation à la pratique d'activité physique et la pratique en elle-même peuvent être initiées ou soutenues grâce à certains dispositifs comme les objets connectés qui, au travers du suivi 
d'activité (e.g., le nombre de pas quotidien), le feedback ou le partage peuvent accroître la satisfaction des besoins fondamentaux.

\section{Les seniors et les objets connectés}

La Silver Economy a tiré profit de la digitalisation en développant une offre visant à aider les seniors à s'informer, surveiller leur santé, suivre correctement leur traitement ou encore à adopter de bons comportements en termes d'activité physique ou de nutrition. Ainsi, si certains seniors sont équipés d'objets connectés de santé (montre, bracelet d'activité mais aussi tensiomètre, balances connectées, pilulier électronique), la très grande majorité d'entre eux possède un smartphone et/ou une tablette et/ou un ordinateur qui leur permettent principalement d'accéder à des contenus adaptés ou encore de communiquer avec leurs proches. Une forte proportion des seniors $(90 \%)$ ont déjà utilisé des outils numériques pour chercher des informations de santé ou encore échanger autour de ce thème ${ }^{11}$.

Une revue de littérature systématique, basée sur le modèle UTAUT de Venkatesh et al. (2003) montre qu'ils apprécient l'aide au quotidien, l'indépendance, la sécurité que les technologies procurent, les possibilités de socialisation et de prise en main de sa santé, mais craignent pour la sécurité de leurs données et la stigmatisation dans le cas d'objets les ciblant spécifiquement. Dans le même temps, ils les considèrent comme trop chers, peu fiables, peu ergonomiques, difficiles à comprendre et trop complexe à utiliser. De plus, ils redoutent que ces objets connectés soient un outil de surveillance au profit d'assurances ou de leur famille (Vassli et Farshchian, 2018). Ils craignent également que les objets connectés les isolent, leur fasse perdre leurs relations sociales. Si l'on fait le lien avec la théorie de l'auto-détermination, il semblerait que, de manière générale, les objets connectés renforcent la satisfaction du besoin d'autonomie, diminue celle du besoin de compétence et d'affiliation (relation aux autres).

Les éléments ci-dessus suggèrent que les seniors sont ambivalents à l'égard des objets connectés, c'est-à-dire qu'ils éprouvent simultanément une attitude positive et négative à leur égard. Ardelet et al. (2017) mettent en évidence - pour la population générale - quatre dimensions qui caractérisent l'ambivalence des personnes vis à vis de ces technologies. Premièrement, la facilité : les objets connectés facilitent et simplifient la vie quotidienne et permettent de gagner du temps, mais ils peuvent aussi être vus comme difficile à comprendre, manipuler et analyser. Deuxièmement, l'intelligence : les objets connectés permettent d'être plus performant, d'être

\footnotetext{
${ }^{11}$ https://toute-la.veille-acteurs-sante.fr/files/2018/06/Communique Seniors e sante et sante connecte e.pdf; Consulté le 20/01/2019.
} 
mieux organisé et d'avoir un contrôle sur sa pratique. Cependant, ils peuvent diminuer le sentiment de compétence et créer une dépendance aux technologies. Troisièmement, le lien social : la communication entre les personnes peut être facilitée mais elles peuvent également ressentir de la confusion autour de ces relations. En effet, une illusion de proximité peut être présente tout en créant un repli sur soi même (isolement). Quatrièmement, l'affect : les objets connectés peuvent procurer certaines émotions et ainsi créer une expérience particulière, qui peut s'avérer positive comme négative, de manière différente selon les individus. Par exemple, la préoccupation pour ses données personnelles (privacy concern) peut disparaître en cas de confiance dans le fournisseur d'objets connectés ou le récipiendaire des données ou encore quand les bénéfices perçus sont élevés. Ceci illustre bien la nécessité de considérer les différences individuelles en termes de perceptions et d'usages. La recherche d'Ardelet et al. (2017) porte sur les objets connectés pour une population générale. A notre connaissance, il n'existe pas de recherche portant explicitement sur l'ambivalence perçue par les seniors et a fortiori faisant explicitement le lien entre leur pratique d'activité physique et l'utilisation d'objets connectés.

$\mathrm{Au}$ final, la revue de littérature suggère que la pratique régulière d'activité physique par les seniors permet de retarder les effets du vieillissement et ainsi, être source de bien vieillir et de bien-être. La motivation autonome semble être un levier majeur pour l'engagement durable dans l'activité physique. Elle pourrait, sous certaines conditions, être soutenue par l'utilisation d'objets connectés, bien que les évidences soient encore peu nombreuses pour les seniors. L’hétérogénéité des pratiques (Pierre et al., 2015), des besoins, des motivations et de leur rapport aux objets connectés, rend essentielle la mise en lumière de profils de seniors afin de personnaliser les leviers de leur engagement dans une activité physique régulière.

\section{Méthodologie}

Compte tenu des objectifs de cette recherche, une approche qualitative a été retenue. Elle repose sur la combinaison d'entretiens semi-directifs (14) et de mini-groupes (2). L'échantillon total se compose de 21 personnes âgées de 63 à 82 ans, toutes retraitées, de Catégorie SocioProfessionnelle variées et ayant des niveaux différents d'activité physique (actuels et dans le passé). La méthodologie est détaillée dans l'encadré 1.

Encadré 1 : Une méthodologie qualitative combinant entretiens individuels et mini-groupes 


\section{Recrutement et échantillon}

Les participants à cette étude ont été recrutés de diverses manières : au travers du réseau personnel d'un des chercheurs par la technique de la « boule de neige » et par le biais d'associations de seniors retraités. Ils ont rempli un questionnaire de "screening". Pour participer à cette étude, il fallait être âgé de 60 ans et plus et être à la retraite. Les données ont été collectées au moyen de deux méthodes complémentaires : 14 entretiens individuels semi-directifs ( 7 femmes et 7 hommes, retraités et âgés en moyenne de 73 ans) et deux mini-groupes ( 3 et 4 personnes, 5 femmes et 2 hommes âgés en moyenne de 71 ans). Au final, l'échantillon compte 21 personnes : 12 femmes et 9 hommes, âgées de 63 à 82 ans, toutes retraitées, de Catégories Socio-Professionnelles variées et ayant des niveaux différents d'activité physique. L'annexe 2 détaille la composition de l'échantillon et explicite les niveaux d'activité physique.

\section{Entretiens individuels semi-directifs}

Lors des entretiens individuels semi-directifs, les thèmes suivants ont été explorés : représentations mentales, motivations et freins des seniors envers l'activité physique et les objets connectés, contribution de l'activité physique et des objets connectés au bien vieillir et au bien-être. Des photos représentant des objets connectés, des personnes pratiquant une activité physique ou de détente, ont été utilisées comme support de la discussion, conformément à la technique de la photo-elicitation.

\section{Mini-groupes}

Les mini-groupes ont été structurés autour de 3 activités : une carte mentale, l'exposition à différentes photos et un jeu de rôle pour explorer les thèmes suivants : représentations collectives et stéréotypes sur l'activité physique et les objets connectés.

\section{Procédure}

Les entretiens ont été menés en face à face dans les locaux d'une université française, pour la plupart. Ils ont été enregistrés en totalité, parfois filmés. Les mini-groupes se sont déroulés dans les mêmes locaux universitaires. Il a été demandé à tous les répondants leur consentement à être enregistrés / filmés et le cas échéant, la signature d'un droit à l'image.

\section{Analyse des données}

Les entretiens et mini-groupes (une trentaine d'heures d'enregistrement au total) ont été entièrement retranscrits. Les données ont fait l'objet d'une analyse de contenu manuelle (horizontale puis verticale). Une grille a été créée de manière émergente à partir des données collectées. Elle a servi de base à la construction du tableau 1. Leur interprétation reflète les allers-retours entre les données, la littérature sur les seniors, la théorie de l'auto-détermination, leur rapport à la technologie en général et aux objets connectés en particulier. L'objectif était d'établir une typologie qualitative de seniors articulée autour de la pratique de l'activité physique, l'auto-détermination et la motivation à la pratiquer en lien avec l'usage des objets connectés, le bien vieillir et le bien-être.

\section{Validité de la recherche}

Conformément aux recommandations de Lincoln et Guba (1985), l'analyse des données a été conduite de manière à maximiser la validité de la recherche. La crédibilité de la recherche est reflétée par la convergence des résultats des analyses menées indépendamment par les chercheurs, sur des données collectées au moyen de deux méthodes (entretien semi-directifs et mini-groupes). Afin de maximiser la fiabilité, la grille de codification comme le codage du corpus ont été réalisés dans un premier temps, par les chercheurs de manière indépendante; puis dans un deuxième temps, les divergences ont été résolues par la discussion. Le taux d'accord inter-codeur mesuré par le coefficient Kappa de Cohen (1960) est supérieur à 80\%. Afin de permettre la transférabilité des résultats, la grille d'analyse ainsi que les différents profils sont clairement explicités. Enfin, dans un souci de confirmabilité, tout au long de l'analyse, les chercheurs ont veillé à l'objectivité de leur analyse par le travail dans un premier temps de manière indépendante et par la discussion entre eux et avec d'autres chercheurs. 
Cohen J. (1960) A coefficient of agreement for nominal scales. Educational and Psychological Measurement, 20: 27-46.

Lincoln Y.S. et Guba EG. (1985) Naturalistic Inquiry, Newbury Park, CA: Sage Publications.

\section{Proposition d'une typologie qualitative de seniors}

L'analyse des données collectées selon la procédure explicitée dans l'encadré 1 a permis la mise en évidence de trois profils de retraités (Tableau 1), fondés sur leur niveau d'activité physique, leur auto-détermination, leur motivation et leur relation aux objets connectés. Le premier groupe comprend 5 répondants, le deuxième en compte 11 et le troisième en comprend 5 (Annexe 2).

\section{Les Contraints}

Les Contraints font peu d'activité physique, ce sont le plus souvent des activités douces (e.g., marche, gym douce, yoga, jardinage, bricolage) : «Moi je fais des exercices d'assouplissement chez moi [...] ça ne veut pas dire je fais deux heures, c'est juste cinq minutes ou dix minutes, juste histoire de.. » (Noureddine, 73 ans). Avant leur retraite, ces pratiques n'étaient pas inscrites dans leur quotidien, ce qui peut justifier le type et le volume des activités pratiquées actuellement : "Ouais moi aussi, enfin non non, pas tout le temps, non quand j'étais jeune, pas grand-chose. Quand j'ai quitté euh pfff, euh non même à la fac je n'en faisais plus non, euh au lycée, jusqu'au lycée c'était obligatoire donc on en faisait, mais après non " (Nuria, 72 ans). Ces activités physiques sont vues avant toute comme une contrainte : «Et puis on nous dit, on n'arrête pas de nous dire que les activités physiques, c'est bien pour lutter contre un tas de choses, contre le diabète » (Nicole 2, 76 ans).

La plupart des Contraints se perçoivent comme faiblement autonomes : ils ne sentent pas à l'origine de leurs choix. De plus, bien souvent, ils n'ont pas le sentiment d'avoir les capacités de mener à bien l'activité physique en raison de leur état de santé : «Non, non, mais moi je me suis fait mal un peu à une jambe là, donc là maintenant, je veux plus, et puis j'ai aussi des problèmes 》 (Nicole 2, 76 ans) ; "Je m'essouffle; même depuis un an c'est très, très sensible, c'est très important et je ne fais presque plus d'activité physique » (André, 82 ans). Leur besoin de compétence n'est donc pas satisfait. Ils pratiquent une activité physique le plus souvent seuls, n'apprécient pas forcément d'être au sein d'un groupe, excepté leur famille ou leurs proches. Leur sentiment d'affiliation, de relation aux autres est donc faible.

Ainsi, la pratique de l'activité physique leur permet une faible satisfaction de leurs besoins psychologiques fondamentaux ce qui les conduit à une motivation externe (ils pratiquent une activité physique pour faire plaisir à leur médecin ou à leur entourage) ou une motivation 
introjectée (se conformer aux pressions extérieures), voire une amotivation : " Moi je ne suis pas comme vous, je n'ai pas de volonté du tout » (Nuria, 72 ans). En d'autres termes, les Contraints présentent une motivation contrôlée voire une amotivation.

La pratique d'une activité physique ne leur procure pas de bien-être. Elle ne joue pas de rôle dans leur bien vieillir. Elle ne leur permet pas de se sentir mieux dans leur vie. D'ailleurs, ils l'abandonnent le plus souvent, assez rapidement. Cela leur permet de se libérer de la contrainte qui pèse sur eux (et donc de retrouver un peu d'autonomie).

Les technologies sont très peu présentes dans leur environnement. Au mieux, ils ont un ordinateur connecté à Internet et un smartphone qu'ils utilisent de manière basique. Ils se sentent peu concernés par les objets connectés : "Alors on est déjà exclu parce que c'est un nouveau mode de vie " (Noureddine, 73 ans), pour ne pas dire perdus et seuls face à eux (manque d'accompagnement dans leur utilisation), "Je trouve que ça va trop vite et moi je suis déjà dépassée par les gens qui ont un iPhone, il y a des petites choses comme ça, j'ai toujours une guerre de retard " (Nicole 2, 76 ans). Les répondants appartenant à ce groupe, n'en voient pas l'utilité : "J'estime que tel que je vis aujourd'hui, c'est pas tout à fait utile » (André, 82 ans) ; soulignant également le côté intrusif de ces technologies perçues comme une réelle menace, «[...] Faut savoir garder un peu de bon sens, parce que là concrètement on est complètement annihilés » (Nourredine, 73 ans). Ils manifestent aussi de la crainte face au traitement et à l'utilisation des données : "Après, ces données elles peuvent aller n'importe où. Alors on vous dit «non non c'est sécurisé ». Mais on n'est pas idiot, même si on n'est pas jeune on n'est pas idiot, on sait très bien que les hackers peuvent s'introduire jusque dans les trucs du Pentagone, alors vous pensez que la déclaration d'impôt, ça va leur poser problème? »(Nuria, 72 ans). Il convient de souligner que bien que ne possédant pas d'objets connectés, quelques répondants dénoncent leur omniprésence dans l'espace public (et tout particulièrement publicitaire) : «Ben parce que si vous êtes allé sur un site de chaussure, 5 minutes après vous avez des chaussures qui vous arrivent sur toutes les pages. Ça change tout le temps d'aspect, ça s'adapte à tout » (Nuria, 72 ans). Au final, ils se sentent peu autonomes, peu compétents face aux objets connectés, ce qui est un frein majeur à leur utilisation.

Les objets connectés, perçus comme peu utiles, ne contribuent pas à la pratique de l'activité physique des Contraints : ils n'utilisent pas leur smartphone pour compter leurs pas que ce soit avec le capteur ou au moyen d'applications dédiées. Etant éloignés de ces objets connectés, les Contraints n'éprouvent donc pas d'ambivalence évidente envers ces derniers.

\section{Les Attentifs}


Les Attentifs pratiquent plusieurs fois par semaine des activités physiques variées : vélo, marche, randonnée, natation, gym douce, yoga, etc. : " Alors vélo, randonnée et marche-promenade 》 (Xavier, 73 ans). Pour la majorité des Attentifs, cette activité physique était déjà présente dans leur quotidien : " J'aimais bien faire du ski de fond, de la danse, course à pied, du yoga » (MarieNoëlle, 68 ans), "J'ai toujours marché, beaucoup, en montagne » (Nicole 1, 75 ans). Ils s'en sentent plutôt capables même si certains soulignent que parfois, ils aimeraient être davantage accompagnés / corrigés pour être sûrs de faire les bons mouvements. Leur niveau de compétence perçu est moyen à fort. Ces activités physiques sont pratiquées presque exclusivement en groupe. Elles sont un moyen de satisfaire un besoin d'affiliation fort : "La gym tout seul, je le ferai pas, hein ? C'est ennuyeux. Tout seul, faire des trucs comme ça, c'est pas très motivant alors qu'en groupe ça a quelque chose de [...]. Le fait d'être en groupe, le fait que l'on force un peu. On est content d'être en groupe, il y a un petit moteur de groupe qui est important 》 (Jacqueline, 66 ans). Leur engagement envers le groupe est un gage de régularité et de plaisir de la pratique. C'est un déterminant fort - et incontournable - de leur motivation à poursuivre la pratique d'une activité physique. Ils ont choisi de s'investir dans ces activités qui sont parfois pratiquées au sein d'un club ou d'une association satisfaisant ainsi leur besoin d'autonomie. Ces activités sont essentiellement réalisées en raison des bienfaits - bien compris - sur le plan de la santé pour la prévention de l'apparition de certains symptômes (Custers et al., 2010) : "Moi je vois bien, si je marche pas un jour ou deux après ben, j'ai des douleurs qui reviennent hein. Et dès que je marche ça passe. Oui oui, faut bouger " (Nicole 1, 75 ans). Les Attentifs manifestent donc une motivation identifiée (i.e., qui présume que l'individu est conscient des bénéfices d'un mode de vie actif; Deci et Ryan, 2000) : «Effectivement je mesure car je suis dans une problématique de vieillissement, je regarde le vieillissement chez les autres et je me dis tiens ça serait bien d'essayer d'entretenir le mieux possible la machine »(Claude, 63 ans). La pratique d'une activité physique avec leurs amis leur procure un bien-être physique, hédonique et mental : «bien dans son corps et dans sa tête » (Paquet et al., 2016) ; "Plaisir. Ah oui oui. Plaisir. Puis rester en forme. Puis j'aime bouger moi j'aime pas rester trop assise. C'est dans mes gênes » (Nicole 1, 75 ans), «Les activités m 'apportent du bien-être, c'est très agréable en plus je bouge je vis mais il y a quelque chose »(Josette, 66 ans), "C'est un équilibre personnel. Absolument. Oui oui » (Monique, 65 ans). La pratique d'une activité physique participe pleinement à leur bien vieillir. Les technologies sont relativement présentes dans l'environnement social des Attentifs : ordinateur connecté à Internet, smartphone, tablette, GPS, etc. Elles sont utilisées pour la recherche d'informations, notamment des avis et conseils d'experts ainsi que pour le maintien du lien avec leurs proches, particulièrement leurs proches qui vivent loin : " Ah oui c'est le lien, je 
pense que oui c'est permanent, on téléphone moins, beaucoup moins et on fait beaucoup plus de message, de mail » (Claude, 63 ans). Ils utilisent principalement des technologies facilitant la communication. En revanche, les objets connectés de santé (montre, tensiomètre, balance) ou domotiques sont peu présents. Les raisons invoquées sont variables. Ils soulignent leur omniprésence. Ils indiquent se sentir oppressés par ces objets connectés et la pression publicitaire afférente. Ils les perçoivent comme une intrusion dans leur vie privée et une atteinte à leur liberté, «Ma liberté c'est moi, certes ma liberté rentre en jeu avec ces objets connectés, j'ai l'impression qu'à ce moment-là elle est un peu restreinte cette liberté » (Josette, 66 ans). Ils rejettent le fait d'être contrôlés : «Ben moi je n'aime pas porter des choses sur moi euh qui sont, sont un peu parasites qui vous permettent de vous situer tout le temps. Euh, t'es où, qu'est-ce que tu fais, quand est-ce que tu rentres ? Bon, ça va quoi» (Monique, 65 ans). Les objets connectés représentent une «menace » pour leur autonomie, ce qui est un frein majeur à leur utilisation. De plus, certains craignent que les objets connectés ne mettent en danger les relations sociales qui sont tellement importantes pour eux : «Pour moi c'est pas un progrès parce que, les gens ils se croisent, et ils sont connectés chacun mais... voilà il y a plus d'échanges quoi » (Jean-Marc, 70 ans). : peu utiles, intrusifs.

Toutefois, les Attentifs reconnaissent « l'intelligence » des objets connectés. " Mais je me suis dit de toute façon, pour avancer, j'ai besoin de nouvelles connaissances, voilà, peut-être que les nouvelles technologies sont là pour m'apporter cette nouveauté" » (Josette, 66 ans) et n'excluent pas que ces technologies notamment axées sur la santé (suivi de leur activité et de leurs paramètres santé) leur soient utiles un jour : «Un jour peut-être que j'y viendrai. Je dis pas non pour moi non plus, je reconnais que c'est un plus, c'est des nouvelles technologies qui sont bien. » [...] «Non, ça me ferait pas peur, je pense pas que ça me ferait peur. Si c'est un mieux pour moi, je l'accepterais. Mais pour l'instant je vois pas véritablement quelle forme ça peut prendre » (Dominique, 67 ans).

Chez les Attentifs, nous retrouvons les dimensions « lien social » et «intelligence » caractéristiques de l'ambivalence. Les membres de ce groupe apprécient les possibilités de communication qu'offrent ces technologies mais ne veulent en aucun cas se sentir contrôlés dans leur utilisation. Leur crainte est que ces objets connectés les isolent et amène une certaine « déshumanisation ».

\section{Les Engagés}

Les Engagés pratiquent de nombreuses activités physiques (e.g., vélo, marche, randonnée, natation, gym douce, yoga, etc.), parfois de manière intense (e.g., cardio, sorties à vélo ou 
randonnées avec un fort dénivelé) et addictive : «Il m’arrive de faire du vélo tous les jours, [...] je fais aussi du tä̈-chi depuis pas mal de temps » (Hervé, 65 ans). Ils veulent rester maîtres des choix liés à leur pratique en termes de fréquence, durée, intensité, moment, endroit. Ils se sentent compétents et autonomes (Dacey et al., 2008). S'ils aiment pratiquer en groupe ou en famille, ils apprécient aussi de le faire seuls. Le groupe n'est pas un facteur déterminant de leur pratique. Au contraire, il est parfois perçu comme un frein à la pratique en toute liberté : par exemple, quand il impose un rythme différent du sien. Ainsi, le sentiment d'appartenance, d'affiliation des Engagés est modéré. Pour certains, la pratique de l'activité physique fait partie du mode de vie qu'ils se sont choisis et reflète qui ils sont : elle relève donc d'une motivation intégrée. Celle-ci implique l'intégration des contraintes externes à son propre système de valeurs (Deci et Ryan, 2000), ce qui amène l'individu à découvrir sa nature profonde (Deci et Ryan, 2016), "C'est un plaisir et un besoin. Les deux »(Pierre, 64 ans). Pour d'autres, la pratique de l'activité physique est vécue comme un défi personnel, une expérience très plaisante : "On se sent tout de suite plus alerte quand on fait du sport c'est évident " (Mustapha, 64 ans). Leur motivation pour l'activité physique est donc intrinsèque (Deci et Ryan, 1985) : faire de l'activité physique, c'est « se sentir vivant " (Hervé, 65 ans). Pour les Engagés, la pratique de l'activité physique est un comportement inscrit durablement dans leur mode de vie : " Du ski, j'ai fait pendant quarante ans, on va dire, du ski de randonnée alpine donc avec les peaux de phoque. J'ai fait du ski de fond, j'ai fait du ski de piste, tous les sports de neige je crois que je les ai tous à peu près utilisés » (Danielle, 71 ans). Elle contribue grandement à leur bien-être physique (se sentir bien dans sa peau), hédonique (se faire plaisir et retirer des satisfactions de la vie) et enfin psychologique ou eudémonique (aligner ses actes et ses valeurs, Ryan et Deci, 2000). L'activité physique leur procure de la vitalité, elle contribue pleinement à leur épanouissement personnel (Ryan et al., 2008) et ainsi à leur bien vieillir : «C'est ça, ce que disait Danielle, entretenir sa santé et entretenir son corps en bon état »(Françoise, 76 ans).

Les technologies sont présentes dans l'environnement social des Engagés. Ils les considèrent comme un réel progrès : «C'est évident que c'est des progrès extraordinaires » (Hervé, 65 ans), et souhaitent en apprendre davantage sur leur utilisation. Ces technologies leur amènent un gain d'autonomie dans leur vie quotidienne mais les rend également plus performants dans leur pratique d'activité physique: «Des fois on aime bien mesurer sa valeur par soi-même, c'est génial moi je dis l'évolution de la science, c'est pour l'évolution des gens » (Mustapha, 64 ans).Les Engagés admettent que ces technologies sont utiles, notamment dans la pratique de leur activité physique en leur permettant d'évaluer leur performances et donc de progresser. Toutefois, leur potentielle entrave à la liberté individuelle est dénoncée : «Oui bien sûr, ça peut 
empêcher la réflexion » (Danielle, 71 ans). Chez les Engagés, nous retrouvons bien les dimensions «facilité » et « intelligence » mises en évidence par Ardelet et al. (2017) avec notamment la peur que ces objets connectés ne rendent l'individu assisté.

Tableau 1 : Caractéristiques des trois profils de seniors.

\begin{tabular}{|c|c|c|c|}
\hline Profils & Contraints & Attentifs & Engagés \\
\hline $\begin{array}{c}\text { Pratique d'activité } \\
\text { physique }\end{array}$ & Très faible à faible & Modérée à assez forte & Forte à très forte \\
\hline $\begin{array}{c}\text { Satisfaction du } \\
\text { besoin d'autonomie }\end{array}$ & Faible & Modérée à assez forte & Forte à très forte \\
\hline $\begin{array}{l}\text { Satisfaction du } \\
\text { besoin de } \\
\text { compétence }\end{array}$ & Faible & Modérée à assez forte & Forte à très forte \\
\hline $\begin{array}{c}\text { Satisfaction du } \\
\text { besoin d'affiliation }\end{array}$ & Faible à très faible & Forte à assez forte & Faible à modérée \\
\hline $\begin{array}{c}\text { Type de motivation } \\
\text { pour l'activité } \\
\text { physique }\end{array}$ & $\begin{array}{l}\text { Amotivation } \\
\text { Motivation externe ou } \\
\text { introjectée (contrôlée) }\end{array}$ & $\begin{array}{l}\text { Motivation identifiée } \\
\text { (autonome) }\end{array}$ & $\begin{array}{c}\text { Motivation intégrée } \\
\text { ou intrinsèque } \\
\text { (autonome) }\end{array}$ \\
\hline Bien-être & Faible & Modéré & Fort \\
\hline $\begin{array}{c}\text { Attitude / } \\
\text { ambivalence vis à } \\
\text { vis des objets } \\
\text { connectés }\end{array}$ & $\begin{array}{l}\text { Utilité pour la collecte } \\
\text { et le partage } \\
\text { d'informations } \\
\text { vs } \\
\text { Menace, intrusion }\end{array}$ & $\begin{array}{c}\text { Pour la prévention et } \\
\text { l'accompagnement vs } \\
\text { Privation de liberté } \\
\text { (social) }\end{array}$ & $\begin{array}{c}\text { Technologie au } \\
\text { service de l'activité } \\
\text { (progression) } \\
\text { vs } \\
\text { Perte de liberté et de } \\
\text { contrôle }\end{array}$ \\
\hline $\begin{array}{c}\text { Place accordée aux } \\
\text { objets connectés en } \\
\text { général }\end{array}$ & Très faible à faible & Modérée à assez forte & Assez forte à forte \\
\hline $\begin{array}{c}\text { Place des objets } \\
\text { connectés dans la } \\
\text { pratique de } \\
\text { l'activité physique }\end{array}$ & Inexistante & $\begin{array}{l}\text { Peu présente car peu } \\
\text { compatible avec le } \\
\text { lien aux autres }\end{array}$ & $\begin{array}{c}\text { Mesure et } \\
\text { accroissement de la } \\
\text { performance }\end{array}$ \\
\hline
\end{tabular}




\section{Quels leviers activer selon les profils de seniors?}

Des recommandations personnalisées pour chaque profil identifié dans cette recherche sont proposées et synthétisées dans le tableau 2.

Favoriser la motivation autonome des "Contraints" et leur acceptation des technologies

Pour les Contraints, les besoins psychologiques fondamentaux d'autonomie, de compétence et d'affiliation ne sont pas ou mal satisfaits, ce qui conduit à l'amotivation ou à une motivation contrôlée et finalement, à peu de bien-être et à un engagement fragile dans l'activité physique (Dacey et al., 2008). Comment aider les seniors de ce groupe à initier la spirale vertueuse de l'activité physique ? Quelle place pour les objets connectés?

Un frein important à la pratique d'activité physique par les Contraints est leur état de santé. Des actions de communication pourraient être menées envers ce groupe afin de leur expliquer que l'activité physique n'est pas synonyme de sport et qu'elle peut s'inscrire dans tous les moments de la journée (ménage, jardinage, marche, etc.), selon des niveaux adaptés à chacun en fonction de leur forme physique. Ces campagnes ciblées pourraient mettre en scène des personnes qui leur ressemblent et qui pour certaines ont les mêmes difficultés, maladies, handicaps qu'elles.

Au plan de la pratique en elle-même, imposer des objectifs d'activité physique, c'est prendre le risque de décourager les Contraints et d'obtenir des résultats contraires. Il conviendrait d'inscrire l'activité physique dans une démarche globale de bien vieillir visant le bien-être des seniors plutôt que l'atteinte d'un objectif quantifié (en nombre de pas, d'escaliers montés / descendus, etc.). En effet, devenant sensibles aux contraintes de l'environnement, ils doivent s'y adapter, et ce au prix d'efforts croissants au fur et à mesure qu'ils avancent en âge. Quand la tension créée par l'écart entre ce qui leur ait demandé et ce qu'ils peuvent / veulent faire devient trop forte, leurs facultés cognitives réduites et leur moins bon état général de santé, associés au sentiment d'être insuffisamment compétents et autonomes, les poussent à privilégier des actions qui leur demandent peu d'efforts (Lawton et Nahemow, 1973), ici en l'occurrence, une faible pratique d'activité physique. Ils peuvent parfois aller jusqu'au désintéressement total de leur environnement et de leur santé. Pour éviter d'en arriver là, il convient de leur redonner en premier lieu du contrôle sur leur vie afin de les amener à se sentir maître et à l'origine de leurs actes mais également libre de s'engager dans un certain comportement. En d'autres termes, il s'agit de les rendre acteurs de leur vieillissement au lieu d'en subir les conséquences et donc de 
pouvoir prendre les décisions qui les concernent, ce qui pourrait par la suite faciliter leur engagement dans des activités physiques quotidiennes (Paquet et al., 2016).

Cela suppose au préalable, que les acteurs proches des seniors (associations, personnel soignant, famille, etc.) identifient leurs besoins, envies, questionnements ou encore aspirations, notamment par rapport à la santé et à l'activité physique. Etre écouté et entendu est un pas vers une meilleure satisfaction de leurs besoins d'autonomie et de compétence. La compréhension de leurs attentes permettrait aussi la mise en place d'un environnement mieux adapté ; un environnement qui les stimulerait et les guiderait, les soutiendrait dans leur engagement dans une activité physique, sans créer une tension contre-productive. La prise en charge par des professionnels de santé (enseignants en APA) qui sauront les écouter, analyser et mettre en œuvre avec eux des activités physiques en adéquation avec leurs envies, besoins et ressources, les aideront à renforcer leur sentiment de compétence en leur procurant des feedbacks personnalisés mais aussi en les aidant à trouver eux-mêmes des solutions pour maintenir cette pratique d'activité physique (Ryan et al., 2008). Les effets attendus sont une réappropriation par le senior de son activité physique et l'expérience de ses bienfaits sur son bien-être, ce qui devrait induire un glissement progressif vers une motivation plus autonome et un engagement plus durable envers la pratique d'une activité physique régulière (Paquet et al., 2016 ; Ryan et al., 2008).

Pour ce qui concerne les technologies du quotidien et particulièrement les objets connectés de santé, la prudence est de mise. En effet, pour la plupart, les Contraints n'en possèdent pas et ne le souhaitent pas car ils n'en voient pas l'utilité, les trouvent trop compliqués Certains les rejettent même en raison de leur caractère intrusif (Vassli et Farshchian, 2018). La première étape serait de leur faire découvrir les objets connectés et particulièrement de santé mais uniquement ceux susceptibles de les aider à bien vieillir et à se sentir bien. Cela supposerait dans un premier temps un accompagnement humain individuel (notamment par la famille) afin de les aider à s'y retrouver dans le foisonnement des offres. En effet, depuis quelques années, la Silver Economy est en ébullition et les offres d'applications mobiles ou d'objets connectés permettant de rester en forme, de se maintenir au domicile ou de surveiller ses paramètres physiologiques en cas de maladie chronique se multiplient. Ces offres nombreuses sont généralement peu adaptées aux besoins spécifiques des Contraints. Elles ne sont pas non plus pensées dans une approche globale du bien vieillir. Elles permettent de répondre à un objectif particulier (géolocalisation, mesure du poids, de la tension, du rythme cardiaque ou autres paramètres physiologiques, rappels de la prise d'un médicament, etc.). Cela supposerait dans un deuxième temps, que les seniors soient accompagnés et aidés dans leur utilisation d'objets connectés pour leur pratique d'activité physique (par exemple par la visualisation de leurs progrès, même minimes) d'autant plus que 
certains peuvent persister dans cette incompréhension malgré des cours d'initiation dédiés aux objets connectés (Vassli et Farshchian, 2018). Il faut donc prendre le temps de leur expliquer les fonctionnalités mais également développer une assistance simple d'accès qui permettrait aux seniors retraités de faire face à leurs difficultés. Il est important qu'ils puissent comprendre et régler eux-mêmes leurs difficultés, renforçant ainsi leur sentiment de compétence et d'autonomie. Cela leur permettrait de faire tomber les barrières associées dans l'UTAUT aux efforts anticipés et de renforcer l'effet positif de l'influence sociale et des conditions facilitatrices sur l'intention. Ainsi, ils pourraient en apprécier l'utilité des objets connectés, un antécédent fort de l'intention d'usage (Venkatesh et al., 2003).

Les Contraints soulignent également un sentiment d'intrusion dans leur vie privée et une absence de contrôle quant à l'utilisation des données. Leur offrir la possibilité de gérer le partage de ces informations devrait les rassurer sur l'utilisation de celles-ci. Les fabricants d'objets connectés pourraient proposer différents «packages » aux seniors, leur offrant la possibilité par exemple d'être les seuls à voir les résultats, de partager ces informations ou encore de définir le nombre de personnes (famille, médecin, référent) pouvant y accéder. Ainsi, ils pourraient décider quelles informations, quel niveau de détails et avec qui les partager. Au final il importe d'apporter des réponses aux barrières cognitives (Venkatesh et al., 2003) mais aussi à l'anxiété exprimée par les membres de ce groupe face aux objets connectés.

\section{Renforcer les compétences et maintenir le lien aux autres des "Attentifs"}

Les Attentifs ont bien compris les bienfaits qu'ils pouvaient retirer de la pratique régulière d'une activité physique. Ils manifestent une motivation identifiée. Ce type de motivation est un bon prédicteur de l'activité physique pratiquée (Gourlan et al., 2013). Elle les aide à persévérer dans leurs efforts. La motivation identifiée va permettre l'installation du comportement dans la durée, accompagné d'un bien-être subjectif et peut être à terme, l'apparition du plaisir dans la pratique (Dacey et al., 2008). On passe alors d'une motivation identifiée à une motivation intrinsèque. Dans les deux cas, la motivation est autonome et susceptible d'induire un engagement durable dans l'activité physique (Ryan et al., 2008).

Pour les Attentifs, c'est principalement la satisfaction du besoin de relation aux autres (Ferrand et al., 2012) qui motive la pratique d'une activité physique et leur engagement. Ainsi, pour les seniors présentant ce profil, les actions de sensibilisation à la pratique d'une activité physique devraient être axées sur l'aspect des liens sociaux que cette pratique peut procurer. A ce niveau, les associations ont un rôle prépondérant à jouer en mettant en relation les seniors autour d'activités physiques qu'ils aiment pratiquer. Elles devraient communiquer de façon à informer 
les seniors sur les possibilités d'activités physiques collectives et surtout sur la possibilité d'essayer ces activités. Les médecins mais aussi les personnels soignants peuvent jouer le rôle d'intermédiaire dans cette communication. En parallèle, les pouvoirs publics et autres acteurs de santé pourraient initier de nouvelles campagnes de communication mettant en scène des seniors heureux de pratiquer en groupe une variété d'activités physiques (à l'instar de ce que font aujourd'hui les assurances et mutuelles).

Les Attentifs sont ambivalents face aux objets connectés : ils leur reconnaissent une certaine utilité (y compris en lien avec l'activité physique) mais dans le même temps, ils sont méfiants et craignent par-dessus tout que leur usage ne soit au détriment des liens aux autres, des moments passés avec des proches. Leur méfiance vient en partie du fait qu'ils ont l'impression de ne pas maîtriser ces technologies. Pour autant, loin de les rejeter, ils sont demandeurs d'aide pour mieux comprendre comment les utiliser et analyser les informations (Vassli et Farshchian, 2018). Il n'en demeure pas moins que leur principal besoin à satisfaire est celui de relation aux autres: c'est à cette condition qu'ils adopteront les objets connectés et les intègreront dans leur quotidien et dans leur pratique d'activité physique. Une clé pourrait être la mise en relation avec d'autres seniors utilisateurs de ces technologies. Ceci leur offrirait la possibilité de partager de façon simple les expériences vécues avec ces objets connectés (et notamment dans le cadre de la pratique d'une activité physique). Egalement, faire des feedbacks et proposer des recommandations personnalisées au travers des objets connectés serait un moyen de répondre à la demande des seniors d'accompagnement pour renforcer leurs compétences et augmenter la valeur perçue de ces objets. Néanmoins, les technologies ne doivent pas être perçues comme une contrainte ou une entrave à la liberté. Cette ambivalence appelle à des actions de sensibilisation, d'information et de formation des seniors sur ces technologies (Vassli et Farshchian, 2018). Dans le but de développer par les Attentifs leur appropriation des objets connectés, il pourrait leur être proposé de participer au processus de co-création de produits qui leur correspondent. L'inclusion de fonctionnalités choisies par eux viendrait renforcer leur utilisation de ces objets connectés cocréés. De plus, la connaissance «de l'intérieur» de l'objet connecté viendrait diminuer les craintes et donc l'ambivalence. La mise en œuvre d'une telle démarche est conforme aux préconisations du marketing social (Gallopel-Morvan, 2014).

\section{Permettre aux « Engagés » de mieux relever leurs défis personnels et se faire plaisir} Les trois besoins fondamentaux des Engagés sont satisfaits, mais c'est avant tout le sentiment d'autonomie couplé à la compétence qui alimente une motivation autonome, des comportements durables et un bien-être eudémonique (Deci et Ryan, 2000 ; Sarrazin et al., 2016). Il est important 
que ces seniors continuent à pratiquer une activité physique régulière pour le plaisir qu'elle leur procure. Il serait intéressant de leur proposer de participer à de nouvelles activités physiques, de façon à stimuler leur curiosité et renforcer leur sentiment de compétence. Organiser davantage de manifestations avec des seniors serait un formidable moyen de partager, transmettre et découvrir diverses expériences.

Les objets connectés doivent les aider à relever leurs défis personnels sans altérer le plaisir de leur pratique ni leur sentiment d'autonomie. Les acteurs de la Silver Economy pourraient en faire des co-créateurs des offres qui leur sont destinées, ce qui les placerait au cœur d'un nouveau projet stimulant. Ils pourraient même devenir des ambassadeurs auprès d'autres seniors, partager leur expérience au quotidien avec ces objets connectés, conseiller et répondre aux difficultés rencontrées par les autres seniors moins familiers avec les technologies.

Tableau 2 : Recommandations par type de profil.

\begin{tabular}{|c|c|c|c|}
\hline Profils & Objectifs & Moyens & Acteurs \\
\hline \multirow[t]{3}{*}{ Contraints } & $\begin{array}{l}\text { Faire comprendre que } \\
\text { l'activité physique } \\
\text { s'inscrit dans une } \\
\text { perspective globale de } \\
\text { bien vieillir au } \\
\text { quotidien }\end{array}$ & $\begin{array}{l}\text { - Campagne de } \\
\text { communication } \\
\text { ciblées } \\
\text { - Information de } \\
\text { proximité }\end{array}$ & $\begin{array}{c}\text { - Pouvoirs publics } \\
\text { - Soignants }\end{array}$ \\
\hline & $\begin{array}{l}\text { Leur redonner du } \\
\text { contrôle sur leur vie et } \\
\text { leur bien vieillir }\end{array}$ & $\begin{array}{l}\text { Les écouter, leur } \\
\text { laisser le choix des } \\
\text { activités qu'ils } \\
\text { souhaitent pratiquer }\end{array}$ & $\begin{array}{l}\text { - Soignants } \\
\text { - Famille }\end{array}$ \\
\hline & $\begin{array}{c}\text { Modifier leur } \\
\text { perception / utilisation } \\
\text { des objets connectés }\end{array}$ & $\begin{array}{l}\text { - Aider au choix } \\
\text {-Favoriser l'essai } \\
\text { - Développer des } \\
\text { offres globales } \\
\text { adaptées au bien } \\
\text { vieillir (produits et } \\
\text { services) }\end{array}$ & $\begin{array}{c}\text { - Famille } \\
\text { - Acteurs de la Silver } \\
\text { Economy }\end{array}$ \\
\hline Attentifs & $\begin{array}{l}\text { - Maintenir ou } \\
\text { renforcer leur niveau } \\
\text { d'activité physique } \\
\text { - Augmenter leur } \\
\text { niveau de compétence }\end{array}$ & $\begin{array}{l}\text { - Campagnes de } \\
\text { communication } \\
\text { ciblées } \\
\text { - Organiser des } \\
\text { rencontres avec des }\end{array}$ & $\begin{array}{l}\text { - Pouvoirs publics } \\
\text { - Associations }\end{array}$ \\
\hline
\end{tabular}


associations de

pratiquants

- Favoriser des

feedbacks sur leurs

activités physiques

\begin{tabular}{|c|c|c|}
\hline $\begin{array}{c}\text { Satisfaire leur besoin } \\
\text { d'affiliation }\end{array}$ & $\begin{array}{l}\text { Organiser des } \\
\text { événements de } \\
\text { proximité permettant } \\
\text { la découverte } \\
\text { d'activité physique à } \\
\text { pratiquer en groupe }\end{array}$ & Associations \\
\hline $\begin{array}{l}\text { Faciliter leur } \\
\text { appropriation des } \\
\text { objets connectés }\end{array}$ & $\begin{array}{c}\text { Leur proposer de } \\
\text { participer au processus } \\
\text { de co-création d'objets } \\
\text { connectés }\end{array}$ & $\begin{array}{c}\text { Acteurs de la Silver } \\
\text { Economy }\end{array}$ \\
\hline $\begin{array}{l}\text { Les accompagner dans } \\
\text { leur recherche de } \\
\text { challenges }\end{array}$ & $\begin{array}{l}\text { Leur proposer de } \\
\text { nouvelles activités }\end{array}$ & Associations \\
\hline $\begin{array}{c}\text { Renforcer leur } \\
\text { utilisation des objets } \\
\text { connectés }\end{array}$ & $\begin{array}{l}\text { - Leur faire découvrir } \\
\text { de nouveaux objets } \\
\text { connectés } \\
\text { - En faire des co- } \\
\text { créateurs et même des } \\
\text { ambassadeurs }\end{array}$ & $\begin{array}{c}\text { Acteurs de la Silver } \\
\text { Economy }\end{array}$ \\
\hline
\end{tabular}

\section{Conclusion}

Cette recherche permet une meilleure compréhension du rapport des seniors à l'activité physique et aux objets connectés. Elle met en évidence une typologie composée de trois profils ayant des besoins psychologiques fondamentaux et des mécanismes de régulation de la motivation différents.

Premièrement, elle montre, conformément à la théorie de l'auto-détermination que la motivation, nourrie par la satisfaction des besoins de compétence, d'autonomie et d'affiliation est un levier de l'engagement dans une activité physique durable pour un plus grand bien-être. Elle contribue ainsi à la théorie de l'auto-détermination et à son application dans le contexte de l'activité physique chez les seniors. C'est une des rares recherches à la mobiliser avec ce public (Paquet et al., 2016). Elle est l'une des toutes premières à faire le lien entre les objets connectés et la pratique d'activité physique. Deuxièmement, elle pose la question du rôle modérateur de l'âge 
(Vassli et Farshchian, 2018; Venkatesh et al., 2003). En effet, dans notre typologie, les répondants les plus âgés n'appartiennent pas tous, contrairement à ce qui aurait pu être attendu, au groupe des Contraints, laissant penser que ce qui compte, c'est non pas l'âge chronologique mais l'âge subjectif (Guiot, 1999). Un décalage entre leur âge chronologique et subjectif incite les individus à mettre en place des stratégies pour contrer les effets de ce dernier (Eraly, 2014) et donc à pratiquer l'activité physique. Troisièmement, elle souligne l'ambivalence (Ardelet et al., 2017) de la majorité des retraités de plus de 60 ans face aux objets connectés, suggérant par le fait même la possibilité, une fois les freins levés et donc l'acceptation des technologies renforcée, de s'appuyer des bénéfices perçus des objets connectés pour amener les seniors vers une motivation autodéterminée favorable à un changement de comportement durable et au final au bien vieillir. A bien des égards, les objets connectés peuvent aider les seniors à se mettre en mouvement et à prendre soin d'eux. Cela passe par une connaissance plus approfondie du rapport qu'ils ont aux objets connectés de santé et par une réflexion sur la manière de les intégrer dans leur environnement, en tant qu'éléments soutenants l'autonomie, la compétence et la relation aux autres (Sarrazin et al., 2016) ; ces objets connectés pouvant in fine, agir comme un levier d'une motivation autonome.

Cette recherche comporte des limites qui tiennent tout d'abord à la taille et la composition de l'échantillon. Ont été interrogées 21 personnes, habitant une zone géographique limitée, retraitées et pour la plupart plutôt en bonne santé, encore assez actives et ouvertes sur la société. Il serait intéressant d'inclure une plus grande diversité de profils en termes de niveau d'activité physique et d'état de santé (Vassli et Farshchian, 2018). Ainsi, des malades présentant des symptômes plus ou moins handicapants pour la pratique d'une activité physique pourraient être interrogés : par exemple des patients atteints de maladies chroniques (e.g., diabète, $\mathrm{BPCO}^{12}$ ), ou encore, des populations vulnérables face aux risques des maladies chroniques en raison de leur mode de vie et de leur environnement social (fumeurs, personnes obèses, habitants des zones défavorisées, etc.). Également, s'entretenir avec des personnes vivant en milieu rural où les structures d'accompagnement à l'activité physique et les équipements sont moins nombreux qu'en ville (Paquet et al., 2016) mais où le mode vie est souvent moins sédentaire, permettrait de dresser un tableau plus complet et d'enrichir la typologie présentée ici. Il serait également intéressant

\footnotetext{
${ }^{12} \mathrm{La}$ bronchopneumopathie chronique obstructive (ou BPCO) est une maladie chronique inflammatoire des bronches. Elle se caractérise par un rétrécissement progressif et une obstruction permanente des voies aériennes et des poumons, entraînant une gêne respiratoire. Elle touche 1,7 millions de personnes en France.

https://www.inserm.fr/information-en-sante/dossiers-information/bronchopneumopathie-chronique-obstructive-bpco ; accédé le 28/09/2018
} 
d'observer si un effet du genre influence la pratique d'activité physique, la satisfaction des besoins fondamentaux, la motivation et le type de régulation et/ou le rapport aux objets connectés de santé (Vassli et Farshchian, 2018).

Cette recherche pourrait utilement être prolongée par une étude dont l'objectif serait dans un premier temps d'observer et de comprendre sur la durée, l'utilisation, l'appropriation et l'intégration dans leur quotidien, par des seniors aux profils variés, d'objets connectés de santé dont ils auraient été équipés. Dans un deuxième temps, il s'agirait de montrer leur effet sur les mécanismes de régulation de la motivation et sur l'activité physique (type et quantité réellement pratiquée à l'aide de l'actimétrie) et au final le bien vieillir dans toutes ses facettes. On disposerait ainsi d'une compréhension des objets connectés comme levier de motivation (autodéterminée) de la pratique d'une activité physique et de leurs effets. Une approche longitudinale permettrait aussi de mettre en évidence l'évolution de la typologie proposée sous l'effet du temps et donc du vieillissement des individus composant les groupes. Par exemple, la question se pose sur l'évolution de la pratique d'activité physique par les Engagés (maintien du nombre et de l'intensité ? jusqu'à quand ?) et du nouveau rôle potentiel joué par les objets connectés qui pourraient venir les soutenir davantage.

Ce champ de recherche à la croisée de l'activité physique, des objets connectés et des seniors est fécond et riche de prolongements sous le double effet de l'évolution des technologies et de l'augmentation de l'espérance de vie (Nunan et Di Domenico, 2019). En effet, avec le vieillissement et malgré une pratique régulière d'activité physique, les capacités réelles des personnes vont diminuer. Relever le défi du maintien d'une motivation autonome, sera encore plus difficile. Il nécessitera une adaptation accrue des stimuli de l'environnement social aux besoins psychologiques fondamentaux des seniors. 


\section{Références bibliographiques}

1. Arbuz G. (2013), Le départ à la retraite : perceptions et accompagnement. Retraite et société $65,168-178$.

2. Ardelet C., Veg-Sala N., Goudey A. et Haikel-Elsabeh M. (2017), Entre crainte et désir pour les objets connectés : comprendre l'ambivalence des consommateurs, Décisions Marketing $86: 31-46$.

3. Barth N., Hupin D., Roche F., Celarier T. et Bongue B. (2018), La prescription de l'activité physique adaptée chez le sujet âgé : de l'intention à la réalité. NPG Neurologie - Psychiatrie - Gériatrie 18 : 155-161.

4. Custers A.F.J., Westerhof G.J., Kuin Y. et Riksen-Walraven M. (2010), Need fulfillment in caring relationships: Its relation with well-being of residents in somatic nursing homes. Aging \& Mental Health 14: 731-739.

5. Dacey M., Baltzell A. et Zaichkowsky L. (2008), Older adults' intrinsic and extrinsic motivation toward physical activity. American Journal of Health Behavior 32: 570-582.

6. Deci E.L. et Ryan R.M. (1985), Intrinsic motivation and self-determination in human behavior. New York: Plenum Press Publishing Co.

7. Deci E.L. et Ryan R.M. (2000), The "what" and "why" of goal pursuits: Human needs and the self-determination of behavior. Psychological Inquiry 11, 227-268.

8. Deci E.L. et Ryan R.M. (2008), Self-determination theory: A macrotheory of human motivation, development, and health. Canadian Psychology/Psychologie Canadienne 49, 182-185.

9. Deci E.L. et Ryan R.M. (2016), Favoriser la motivation optimale et la santé mentale dans les divers milieux de vie, Ch. 2 : 15-32. In C. Martin Krumm et C. Tarquinio (Eds.), Traité de psychologie positive, Bruxelles : De Boeck.

10. Eraly H. (2014), Marketing senior and young-retirees' resistance to aging. Émulations : Revue Des Jeunes Chercheuses et Chercheurs En Sciences Sociales 7, 69-82.

11. Ferrand C., Nasarre S., Hautier C. et Bonnefoy M. (2012), Aging and well-being in French older adults regularly practicing physical activity: A self-determination perspective. Journal of Aging and Physical Activity 20, 215-230.

12. Franco M.R., Tong A., Howard K., Sherrington C., Ferreira P.H., Pinto RZ. et Ferreira M.L. (2015), Older people's perspectives on participation in physical activity: A systematic review and thematic synthesis of qualitative literature. British Journal of Sports Medicine 49, 12681276. 
13. Gallopel-Morvan K. (2014), Marketing social et marketing social critique : quelle utilité pour la santé publique? Les Tribunes de la santé 45, 37-43.

14. Gillet N. et Vallerand R.J. (2016), Les effets de la motivation sur la performance sportive au regard de la théorie de l'autodétermination: vers une approche intra-individuelle. Psychologie Française 61, 257-271.

15. Gourlan M., Sarrazin P. et Trouilloud D. (2013), Motivational interviewing as a way to promote physical activity in obese adolescents: A randomised-controlled trial using selfdetermination theory as an explanatory framework. Psychology and Health 28(11), 12651286.

16. Guiot D (1999), Age subjectif et segmentation des seniors. Décisions Marketing 18, 15-23.

17. Gurviez P et Sirieix L. (2017), Marketing et bien-être : un objectif complexe, Décisions Marketing, 87, Numéro spécial « Marketing et bien-être », Editorial.

18. Lawton M.P. et Nahemow L. (1973), Ecology and the aging process, in: The Psychology of Adult Development and Aging. American Psychological Association, Washington, DC, US, 619-674.

19. Nunan D. et Di Domenico M. (2019), Older consumers, digital marketing, and public policy: A review and research agenda, Journal of Public Policy \& Marketing 38(4), 469-483.

20. Mareschal J et Genton L. (2014), Activité physique et santé chez la personne âgée : évidences et recommandations. Nutrition Clinique et Métabolisme 28, 263-271.

21. Paquet Y., Junot A., David M. et Vallerand R.J. (2016), Vieillissement réussi : entre contrôle et autonomie, le bon équilibre motivationnel, Ch. 10, 193-208, In Paquet C, Carbonneau N et Vallerand R.J (Eds.), La théorie de l'autodétermination. Aspects théoriques et appliqués, Louvain-la-Neuve, De Boeck.

22. Pierre J., Caluzio C. et Schut P.O. (2015), La pratique sportive des seniors : des profils et besoins variés. Retraite et société, Caisse nationale d'assurance vieillesse (CNAV) / La Documentation Française, 75-90.

23. Ryan R., Patrick H., Deci E. et William G. (2008), Facilitating health behaviour change and its maintenance: Interventions based on Self-Determination Theory. European Psychologist, $10,2-5$.

24. Sarrazin P., Cheval B. et Isoard-Gautheur S. (2016), La théorie de l'autodétermination : un cadre pour comprendre et nourrir la motivation autonome dans le domaine de l'activité physique pour la santé et du sport, Ch. 14, 270-292, In Paquet C, Carbonneau N et Vallerand RJ (Eds.), La théorie de l'autodétermination. Aspects théoriques et appliqués, Louvain-laNeuve : De Boeck. 
25. Sengès E., Guiot D. et Chandon J.L. (2018), Le Bien Vieillir Désiré : quelle validité prédictive auprès des consommateurs âgés de 50 à 80 ans ? Recherche et Applications en Marketing 1(34), 3-24.

26. Singleton J. (2017), Foreword: Role of leisure and physical activity for Millennials, Generation X (seniors/older adult in training), and current cohort of seniors. Topics in Geriatric Rehabilitation 33(3), 153-155.

27. Teixeira P.J., Carraça E.V., Markland D., Silva M.N. et Ryan R.M. (2012), Exercise, physical activity, and self-determination theory: A systematic review. International Journal of Behavioral Nutrition and Physical Activity, 9, 78.

28. Urien B. (2014), Comprendre le vieillissement, un enjeu économique et académique vital. Décisions Marketing, 76, 7-11.

29. Vassli L.T. et Farshchian B.A. (2018), Acceptance of health-related ICT among elderly people living in the community: A systematic review of qualitative evidence. International Journal of Human-Computer Interaction, 34(2), 99-116.

30.Venkatesh, V., Morris, M., Davis, G., et Davis, F. (2003). User acceptance of information technology: Toward a unified view. MIS Quarterly, 27(3), 425-478. 


\section{Annexes}

\section{Annexe 1 : La motivation dans la Théorie de l'Auto-Détermination}

La théorie de l'évaluation cognitive (Cognitive Evaluation Theory), une des sous-théories de la Théorie de l'Auto-Détermination (SDT), identifie six types de motivations qui se répartissent (qualitativement) sur un continuum allant de l'amotivation (absence de motivation) à la motivation intrinsèque (niveau maximum d'auto-détermination) en passant par la motivation extrinsèque (niveau intermédiaire d'autodétermination). On parle d'amotivation quand la personne n'éprouve aucune sorte de motivation. La motivation extrinsèque renvoie à la pratique d'un certain comportement pour atteindre un résultat qui n'est pas en rapport avec le comportement en lui-même (récompense, éviter une punition). Cette motivation extrinsèque se divise en quatre types de motivation selon le type de régulation :1) la régulation externe où la personne va agir uniquement pour obtenir une récompense ou éviter une punition, 2) la régulation introjectée où le comportement est réalisé en réponse à des pressions internes ou externes (sentiment de culpabilité, menace envers l'estime de soi), 3) la régulation identifiée où les activités sont réalisées car elles sont considérées comme importantes et possèdent un sens profond pour la personne à la suite d'une prise de conscience et 4) la régulation intégrée sera identifiée chez une personne qui réalise une activité pour découvrir sa nature profonde. Enfin, dans la motivation intrinsèque, la personne réalise une activité pour le plaisir qu'elle lui procure. Les régulations externe et introjectée correspondent à des motivations contrôlées alors que les motivations identifiée, intégrée et intrinsèque sont des motivations autonomes.

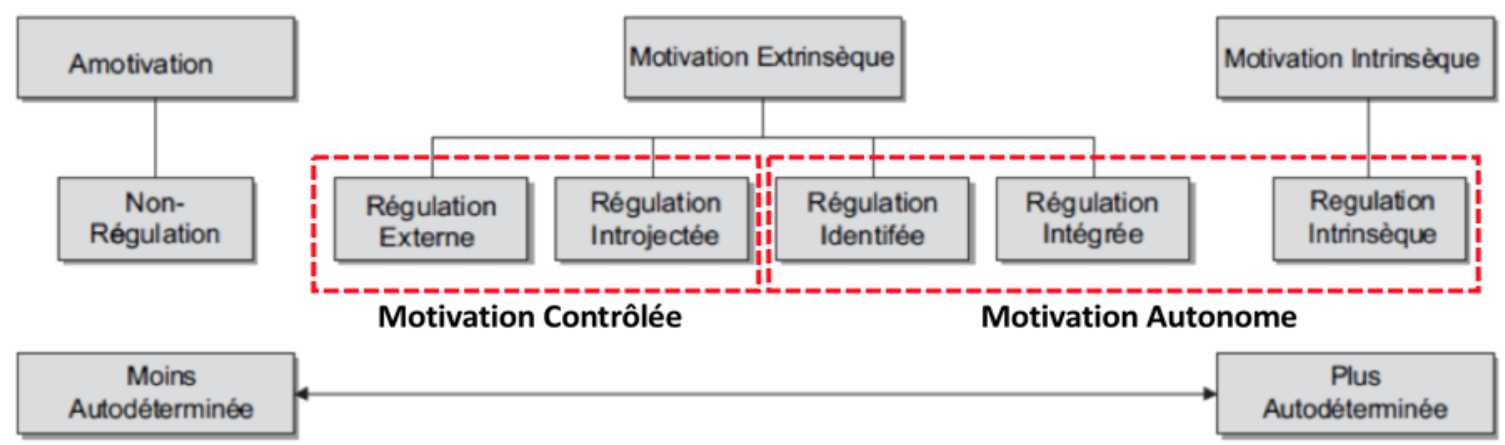

(Adapté de Deci et Ryan, 2000) 
Annexe 2 : Caractéristiques de l'échantillon et appartenance à la typologie

\begin{tabular}{|c|c|c|c|c|c|c|c|}
\hline Prénom & $\begin{array}{c}\text { Genre } \\
\text { (Femme/ } \\
\text { Homme) }\end{array}$ & $\begin{array}{c}\text { Age } \\
\text { (années) }\end{array}$ & $\begin{array}{l}\text { Profession avant } \\
\text { la retraite }\end{array}$ & $\begin{array}{c}\text { Niveau } \\
\text { antérieur } \\
\text { d'AP }\end{array}$ & $\begin{array}{c}\text { Niveau } \\
\text { d'AP } \\
\text { actuel ** }\end{array}$ & $\begin{array}{c}\text { Place } \\
\text { des OC } \\
* * *\end{array}$ & Profil \\
\hline Alain & $\mathrm{H}$ & 68 & Peintre décorateur & ++ & + & + & Contraints \\
\hline André & $\mathrm{H}$ & 82 & $\begin{array}{c}\text { Chargé de } \\
\text { développement }\end{array}$ & ++ & + & + & Contraints \\
\hline Chantal & $\mathrm{F}$ & 65 & Traductrice & ++ & ++ & ++ & Attentifs \\
\hline Claude & $\mathrm{H}$ & 63 & Informaticien & ++ & ++ & ++ & Attentifs \\
\hline Danielle & $\mathrm{F}$ & 71 & Bibliothécaire & +++ & +++ & +++ & Engagés \\
\hline Dominique & $\mathrm{F}$ & 67 & Enseignante & ++ & ++ & ++ & Attentifs \\
\hline Françoise & $\mathrm{F}$ & 76 & Formatrice & +++ & +++ & +++ & Engagés \\
\hline Hervé & $\mathrm{H}$ & 65 & $\begin{array}{l}\text { Responsable } \\
\text { d'équipe }\end{array}$ & +++ & +++ & +++ & Engagés \\
\hline Jacqueline & $\mathrm{F}$ & 66 & Enseignante & ++ & ++ & ++ & Attentifs \\
\hline Jean-Marc & $\mathrm{H}$ & 70 & Ingénieur & + & ++ & + & Attentifs \\
\hline Josette & $\mathrm{F}$ & 66 & Enseignante & + & ++ & ++ & Attentifs \\
\hline Maria & $\mathrm{F}$ & 69 & Infirmière & + & ++ & + & Attentifs \\
\hline $\begin{array}{l}\text { Marie- } \\
\text { Noëlle }\end{array}$ & $\mathrm{F}$ & 68 & Médecin & ++ & ++ & + & Attentifs \\
\hline Monique & $\mathrm{F}$ & 65 & $\begin{array}{l}\text { Conservatrice de } \\
\text { bibliothèque }\end{array}$ & ++ & ++ & + & Attentifs \\
\hline Mustapha & $\mathrm{H}$ & 64 & Bibliothécaire & ++ & ++ & +++ & Engagés \\
\hline Nicole 1 & $\mathrm{~F}$ & 75 & Garde d'enfants & ++ & ++ & + & Attentifs \\
\hline Nicole 2 & $\mathrm{~F}$ & 76 & Assistance sociale & + & + & + & Contraints \\
\hline Noureddine & $\mathrm{H}$ & 73 & Ingénieur & + & + & + & Contraints \\
\hline Nuria & $\mathrm{F}$ & 72 & Enseignante & + & + & + & Contraints \\
\hline Pierre & $\mathrm{H}$ & 64 & Commercial & +++ & +++ & ++ & Engagés \\
\hline Xavier & $\mathrm{H}$ & 73 & $\begin{array}{c}\text { Employé de la } \\
\text { grande } \\
\text { distribution }\end{array}$ & ++ & ++ & + & Attentifs \\
\hline
\end{tabular}

* Codification en trois niveaux du niveau d'activité physique (AP) passé :

+ : Chez ce groupe, la pratique régulière d'AP n'était pas présente ou bien effectuée de manière discontinue.

++ : Les personnes de ce groupe ont pratiqué régulièrement des activités de type course, vélo ou randonnée. 
+++ : Les personnes de ce groupe pratiquent également diverses activités et déclarent avoir essayé nombre d'entre elles.

** Codification en trois niveaux de l'activité physique (AP) :

$+:$ Ce groupe présente un faible niveau d'activité physique. Dans cette étude, les participants ne rapportent généralement qu'une seule activité. Ce sont des activités douces (marche, assouplissement) qui souvent ne sont pas réalisées quotidiennement.

++ : Ce groupe réalise plusieurs activités physiques (marche, gym, escalade) et ceci plusieurs fois par semaine.

+++ : Ce groupe pratique plusieurs activités physiques de façon plus intensive (vélo, ski, aquagym). Ces activités sont également couplées à des activités plus douces (randonnée, bricolage, jardinage).

*** Codification en trois niveaux de la place accordée aux objets connectés (OC)

+ : Les objets connectés sont quasi absents de leur vie. Ils possèdent tout au plus un téléphone, un ordinateur ou une tablette. Leur utilisation est très réduite en raison du rejet de ces technologies dont ils ne perçoivent pas l'utilité.

++ : Les objets connectés sont présents dans leur vie. Ils les utilisent régulièrement pour des tâches bien définies (communication, information). Certains d'entre eux reconnaissent leur «intelligence» et font des efforts pour essayer de les maîtriser.

+++ : Ce groupe est plus ouvert aux objets connectés, en possède plusieurs (dont des objets connectés de santé et de bien-être). Ils les utilisent régulièrement et les considèrent comme un réel progrès. 